\title{
Tantalum strength at extreme strain rates from impact-driven Richtmyer-Meshkov instabilities
}

\author{
Michael B. Prime $\odot,{ }^{1}$ William T. Buttler, ${ }^{1}$ Saryu J. Fensin, ${ }^{1}$ David R. Jones $\odot,{ }^{1}$ Justin L. Brown, ${ }^{2}$ Robert S. King $\odot,{ }^{1}$ \\ Ruben Manzanares, ${ }^{1}$ Daniel T. Martinez $\odot,{ }^{1}$ John I. Martinez, ${ }^{1}$ Jeremy R. Payton, ${ }^{1}$ and Derek W. Schmidt ${ }^{1}$ \\ ${ }^{1}$ Los Alamos National Laboratory, Los Alamos, New Mexico 87545, USA \\ ${ }^{2}$ Sandia National Laboratories, Albuquerque, New Mexico 87185, USA
}

(Received 7 August 2019; published 6 November 2019)

\begin{abstract}
Recently, Richtmyer-Meshkov instability (RMI) experiments driven by high explosives and fielded with perturbations on a free surface have been used to study strength at extreme strain rates and near zero pressure. The RMI experiments reported here used impact loading, which is experimentally simpler, more accurate to analyze, and which also allows the exploration of a wider range of conditions. Three experiments were performed on tantalum at shock stresses from 20 to $34 \mathrm{GPa}$, with six different perturbation sizes at each shock level, making this the most comprehensive set of strength-focused RMI experiments reported to date on any material. The resulting estimated average strengths of $1200-1400 \mathrm{MPa}$ at strain rates of $10^{7} / \mathrm{s}$ exceeded, by $40 \%$ or more, a common power law extrapolation from data at strain rates below $10^{4} / \mathrm{s}$. Taken together with other data in the literature that show much higher strength at simultaneous high rates and high pressure, these RMI data isolated effects and indicated that, in the range of conditions examined, the pressure effects are more significant than rate effects.
\end{abstract}

DOI: 10.1103/PhysRevE.100.053002

\section{INTRODUCTION}

The ability of viscosity to reduce the growth rate of Richtmyer-Meshkov instabilities (RMI) in fluids has been studied for some time [1-5]. The sensitivity of RMI to strength in solids, the analog of viscosity, has received explicit attention more recently [6-12] with increasing attention also in regard to ejecta [13-16]. Since the proposal about a decade ago to use RMI to evaluate strength $[17,18]$, several experimental efforts to use this capability have been reported [19-29], although in many cases the experiments serve as validation experiments for strength models rather than providing quantitative estimates of strength.

This work builds and improves upon previous efforts that also capitalized on the more experimentally accessible freesurface (Atwood number $=-1$ ) RMI configuration to quantitatively estimate strength at low pressure and rates up to at least $10^{7} / \mathrm{s}$ [19-22]. The previous efforts used high explosive loading to generate the shock. This work reports RMI strength measurements using impact loading to improve the process. Estimating strength from RMI data requires modeling the experiments in a hydrocode or something similar. Impact loading can be modeled both more simply and more accurately than high explosive loading, as illustrated by the significant previous effort to model explosive loading [22]. More accurate modeling should lead to easier and more accurate strength estimation. Furthermore, impact loading makes it simpler to

Published by the American Physical Society under the terms of the Creative Commons Attribution 4.0 International license. Further distribution of this work must maintain attribution to the author(s) and the published article's title, journal citation, and DOI. experimentally adjust impact pressure and interrogate strength over a wider range of conditions.

Figure 1 illustrates a typical RMI experiment for strength in the free-surface configuration. The sample is impacted on the face opposite machined sine wave perturbations. The initial perturbation size is characterized by the nondimensional number $\eta_{0} k$ (where $k=2 \pi / \lambda$ ). The perturbations start to invert after shock, and the subsequent peaks that started as low spots are called spikes. Depending on the perturbation size, the shock magnitude, and the strength of the material the perturbation may arrest or may grow unstably. In the traditional fluid mechanics understanding, the original high spots invert and become low spots called bubbles. In the experiments on high strength solids in this work, the inversion does not progress that far in the time of interest, so Fig. 1 does not show traditional bubbles.

\section{EXPERIMENTS}

This study used three tantalum targets, each with six separate regions of perturbations ranging in size from $\eta_{0} k=0.3$ to 0.9 . The targets were impacted in a gas gun with a tantalum impactor at different impact velocities, producing stresses of approximately 20,30, and $34 \mathrm{GPa}$. Time resolved velocities of the perturbations were measured. The details follow.

\section{A. Sample fabrication and inspection}

The experimental targets were made from the same batch of annealed Starck tantalum that has been well characterized and used in other work [30-32]. The average grain size is about $25 \mu \mathrm{m}$, and the plate material was carefully clock rolled to produce minimal texture and anisotropy. The three nominally identical three-piece tantalum targets shown in 


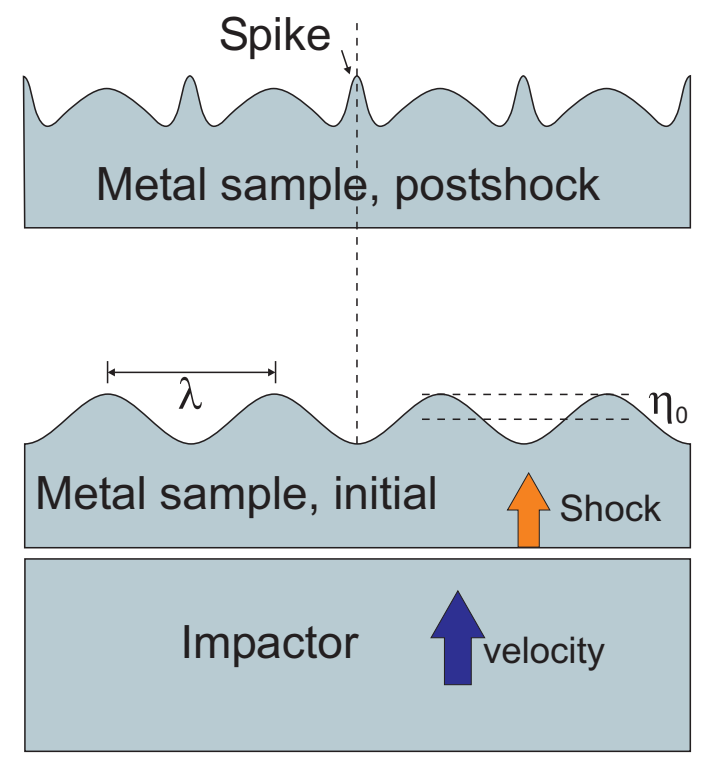

FIG. 1. Sketch of a free-surface Richtmyer-Meshkov instability experiment. The bottom figure is the initial condition and the top is postshock. Perturbation amplitudes are exaggerated.

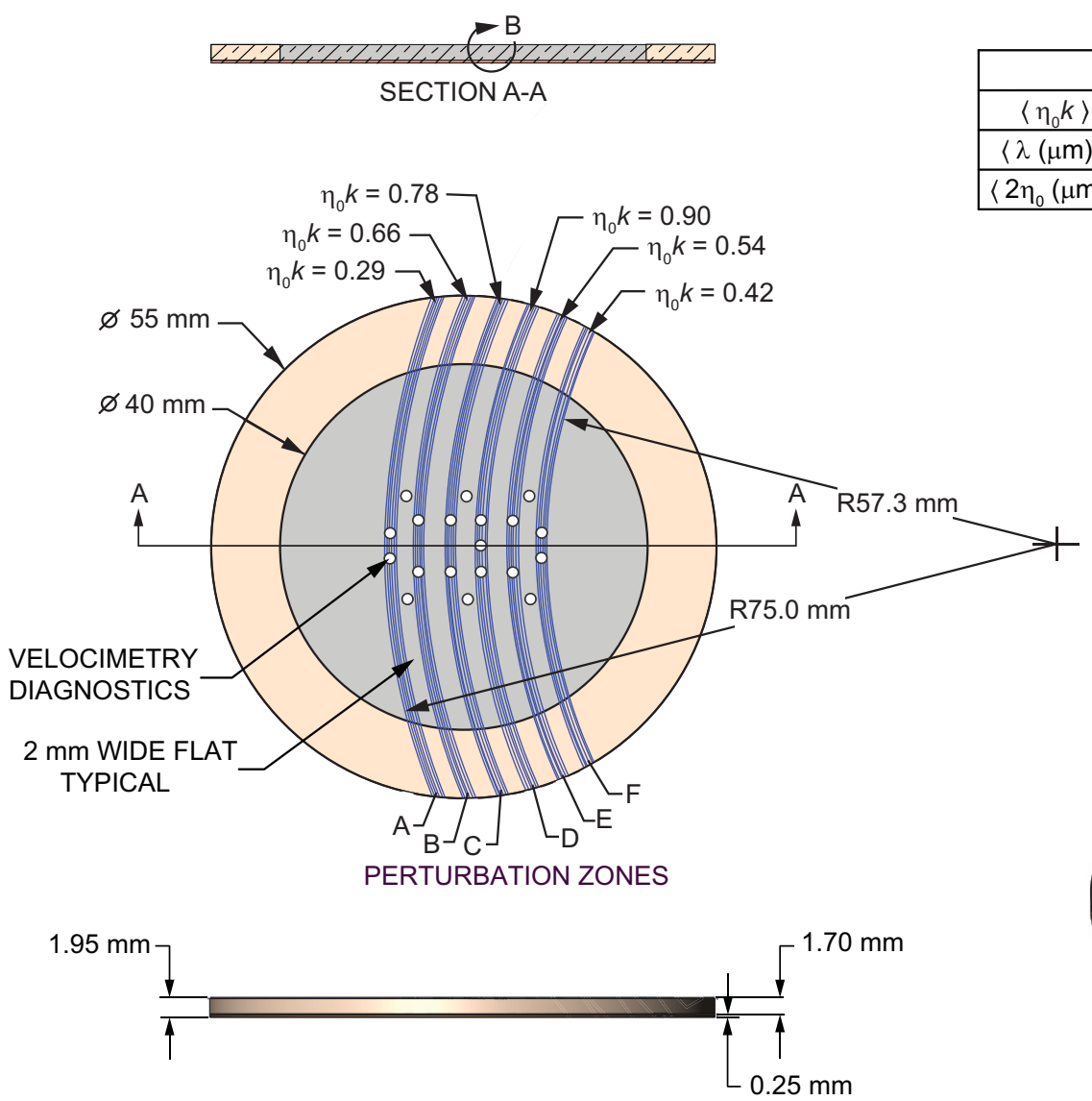

Fig. 2 consist of a 40-mm diameter and 1.70-mm-thick disk of Starck Ta press fit into a 55-mm-diameter momentum trapping ring of common (not Starck) Ta, with both then epoxied with AngströmBond to a 0.25 -mm-thick common Ta foil to hold the pieces together. The assembled target was machined with six regions each containing 5 wavelengths of a sinusoidal surface perturbation machined along the full arc length shown in Fig. 2. Each region had a constant $\eta_{0} k$, which ranged from 0.3 to 0.9 over the six regions. The wavelengths were nominally $0.25 \mathrm{~mm}$ except for the $\eta_{0} k=0.9$ region, which had a wavelength of $0.3 \mathrm{~mm}$ because of machining limitations. The machining details are discussed below.

Machining the targets proved challenging because of the hardness of the Ta. Previously, our RMI metal-strength targets were machined by mounting a diamond tipped tool, at a radius of $r \geqslant 150 \mathrm{~mm}$, to the spindle of an air-bearing lathe. In this situation, the rough-cut metal target was mounted onto a tool post on the lathe bed. Spinning the tool on the lathe's air-bearing spindle in a fly-cutter operation gives a mirrorlike surface finish on the metal target, with perturbations being machined as the cutter translates in and out.

The Ta targets in this study required a unique approach. Initial tests in the fixed radius fly-cutting geometry failed

\begin{tabular}{|c|c|c|c|c|c|c|}
\multicolumn{1}{c|}{} & \multicolumn{6}{c|}{ AVERAGE QUANTITIES } \\
\cline { 2 - 7 } \multicolumn{1}{c|}{} & \multicolumn{6}{c|}{ PERTURBATION ZONE } \\
\hline & A & B & C & D & E & F \\
\hline$\left\langle\eta_{0} k\right\rangle$ & 0.289 & 0.671 & 0.788 & 0.895 & 0.548 & 0.420 \\
\hline$\langle\lambda(\mu \mathrm{m})\rangle$ & 247.8 & 247.5 & 246.9 & 297.6 & 248.5 & 249.1 \\
\hline$\left\langle 2 \eta_{0}(\mu \mathrm{m})\right\rangle$ & 22.7 & 52.9 & 61.9 & 84.8 & 43.4 & 33.3 \\
\hline
\end{tabular}

FIG. 2. Each target was made from three pieces of tantalum and had six regions with different perturbation amplitudes. Laser Doppler velocimetry locations are denoted as white circles on the six perturbation regions, and three flat regions. Within each region, the probes are numbered consecutively starting at 1 at the bottom of this figure. 


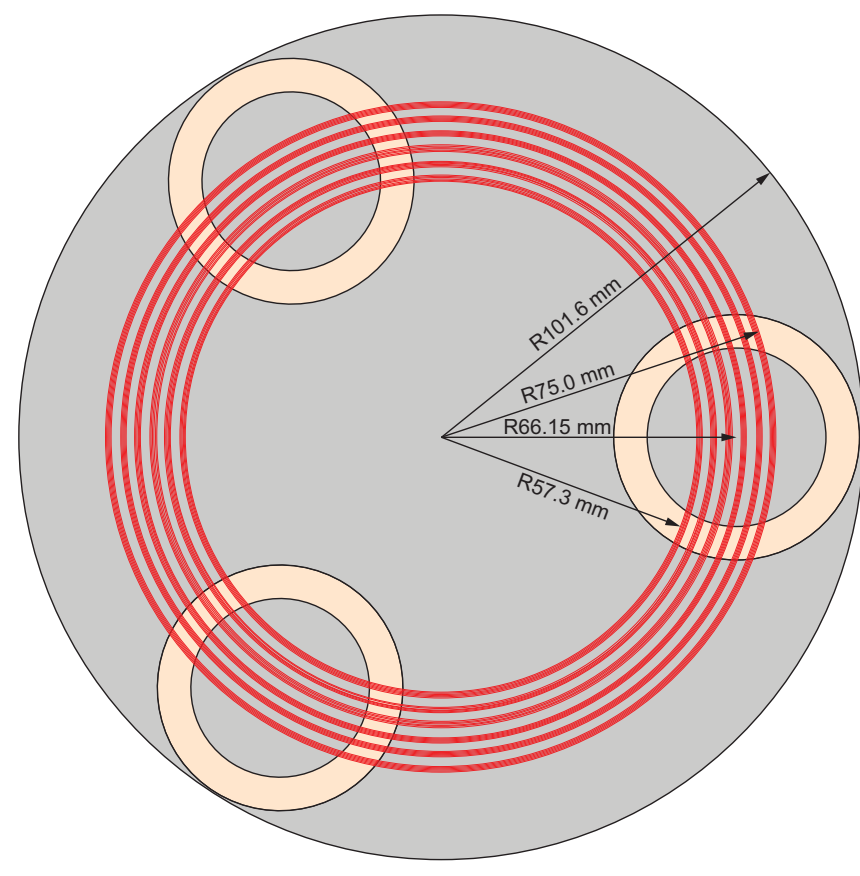

FIG. 3. The three rough-cut and press-fitted targets were glued into a larger Ta plate. Subsequently, the targets and plate were turned on an air-bearing lathe to generate nominally identical profiles on all three targets, as shown in Fig. 2.

as the tools wore down quickly with the interrupted tool cuts. Other machining tests with wire electrical discharge machining also failed as the wire "burned" diameters were too large to achieve the desired wavelengths and amplitudes of the Ta target perturbations. Subsequently, we devised the approach shown in Fig. 3 where the three rough-cut sample assemblies were epoxied with Loctite into pockets machined into a 203-mm-diameter Ta plate, which was then mounted to the spindle of the air-bearing lathe. A carbide tipped tool was then mounted at a $30^{\circ}$ positive rake angle onto the tool post and then radially translated and indexed in and out as

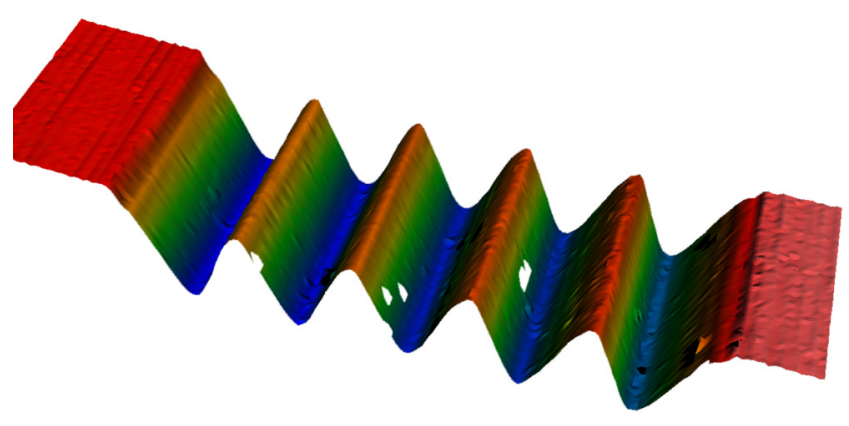

(a) the spindle rotated at $800 \mathrm{rpm}$ to cut the perturbation shapes into the Ta target. In contrast to our fixed radius targets, the perturbation radii vary. Each 5-micron depth-of-cut pass across the surface consumed three tools. After a tool was consumed, the new tool had to be reindicated.

Once the targets were finished, they were removed from the larger Ta plate with Loctite remover and the final profiles for each perturbation region in each target were scanned with both an OGP optical coordinate measuring machine using an interferometer laser and a Zygo white light interferometer. Figure 4 shows results for the $\eta_{0} k=0.54$ region on the 20 $\mathrm{GPa}$ target. Most of the results are of similar or better quality, with the exception that the $\eta_{0} k=0.3$ regions tended to have somewhat rougher profiles. Figure 4(b) shows that the profile fits a sine wave quite well. Such fits were used to determine the as-built perturbation geometry for each region, given in the table later in the Results section.

\section{B. Velocimetry}

As seen in Fig. 2, each experiment included 19 photon Doppler velocimetry (PDV) [33,34] probes aligned normal to the target surface: six on flat regions, and thirteen on perturbation regions. A narrow linewidth 1550-nm-wavelength fiber laser was sent to the PDV probes, illuminating the target surface under the probe. The light reflected back from the target was collected by the same probe, and isolated using a circulator. If the target surface is moving, the collected reflected light will be Doppler shifted. This shifted light was then mixed with a reference wavelength, typically a few tens of picometers offset from the source wavelength, to produce a beat frequency proportional to the surface velocity. This beat frequency was recorded on high bandwidth oscilloscopes, and short-time Fourier transform techniques were used to reduce the power spectrum for each probe. In these experiments, where the PDV probe is imaging a surface with a continuum of velocities in the field of view, the spectrograms contained a power spectrum corresponding to all of the velocities. This ability of PDV to track multiple velocities makes it an ideal

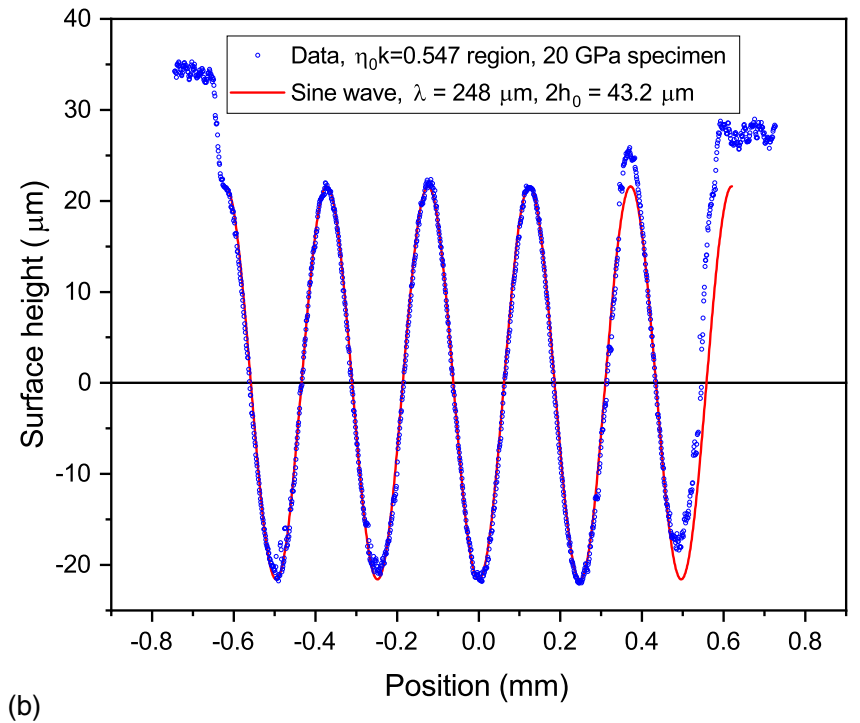

FIG. 4. (a) Typical inspection data on a perturbation region show a continuous profile that (b) generally fits a sine wave profile quite well. 
diagnostic for RMI experiments. As the amount of reflected light collected can vary by several orders of magnitude during the experiment, due to the change in surface profile in the perturbed regions, the signal from each PDV photodetector was split into two oscilloscope channels, one on a high sensitivity (volts per division) and one on a low sensitivity setting. Hence, if a sudden increase in light collection occurs and the high sensitivity channel saturates, the low sensitivity data can be used. Since Fourier transforms are used to reduce the data, saturating a channel generates harmonics and makes calculating the velocity history difficult.

Given the cost of high bandwidth and high sample rate scopes, some PDV signals were upshifted or frequency multiplexed so multiple signals could be recorded on a single channel. While these systems reduce the cost per signal, the trade-off is some increase in noise and reduced bandwidth. For these reasons, we record at least one signal from each perturbation zone on individual scopes with the high and low voltage coverages, and we upshifted and multiplexed the flat velocimetry and impactor velocity. Some of the duplicate signals on perturbation zones were upshifted and one was frequency multiplexed.

The $1 / e^{2}$ diameter of the collimating probes used for PDV was $0.3 \mathrm{~mm}$. Repeated trials of mounting probes showed that the laser spots were aligned on the center of each perturbation region to within $\lambda / 2$. This precision means that the PDV probe might see any of the three central spikes but not the outer two. Maximum deviation of the probes from surface normal was $8^{\circ}$ with most much closer to normal. Since the cosine of $8^{\circ}$ is 0.99 , the maximum error in velocity is $1 \%$. Since that is the maximum error and the error should vary randomly from probe to probe, the influence on the overall results is expected to be minimal.

\section{Execution}

Each target was bonded to a Lexan plate to mount the target in a gas gun. The plate had a three-dimensional (3D) printed attachment to position the $19 \mathrm{PDV}$ probes $40 \mathrm{~mm}$ above the surface as shown in Fig. 5. One more PDV probe was mounted in the Lexan plate and collimated down the gas-gun barrel to measure the impact velocity. A single piezoelectric pin was also mounted in the Lexan plate to provide a trigger signal for the diagnostics at impact. A series of irises, mirrors, and a HeNe laser was used to align the Lexan plate normal to the axis of the gas-gun barrel to provide a planar impact. Typical impact tilts achieved with this technique are submilliradian.

For all three experiments, the flyer plate was a $38-\mathrm{mm}-$ diameter, 2.5-mm-thick Ta disk. This was mounted to the front of the projectile, supported by a glass microbead material to avoid bowing of the flyer plate during the launch. An 80 -mm-diameter gas gun was used to accelerate the projectile to velocities from $\sim 640$ to $\sim 1000 \mathrm{~m} / \mathrm{s}$, generating stresses of approximately 20, 30, and $34 \mathrm{GPa}$.

\section{ANALYSIS APPROACH AND MODELING}

As in previous approaches in the literature, this work estimates an average von Mises effective strength, $Y$, by using a model and finding the constant strength that best matches the

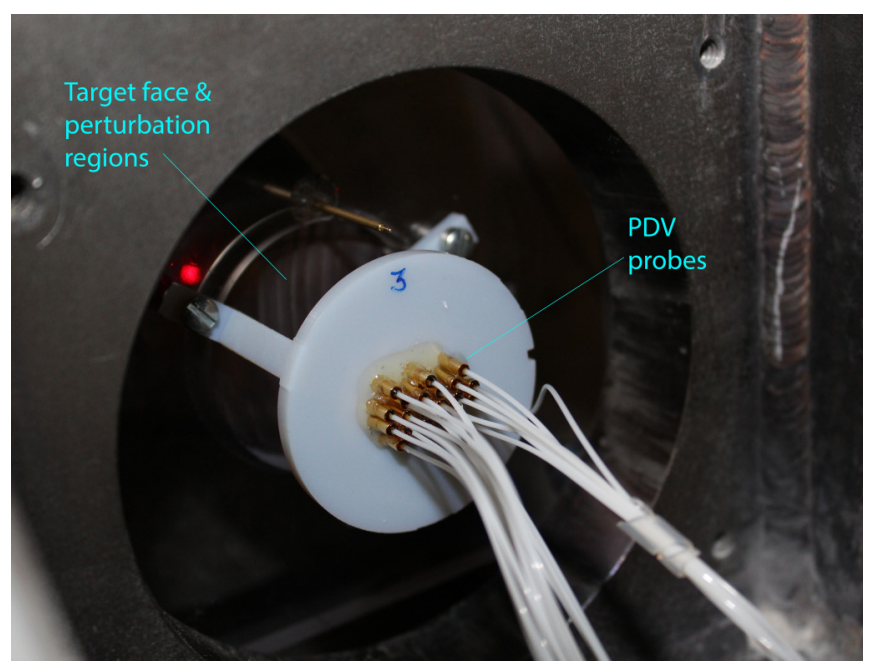

FIG. 5. A view of the target free surface and the PDV probes prior to execution.

data. The utility of an average strength depends on the hypothesis that the RMI experiment exercises a fairly compact range of the independent variables that affect strength: strain, strain rate, temperature, and pressure. Preliminary work supports the compactness notion for RMI [35], and Ta shows modest strain hardening at high rates $[30,36]$ and modest or negligible shock hardening [37], strengthening the argument for a meaningful average strength. However, further work is needed to fully demonstrate the usefulness of the average strength approach.

To estimate strength, this work uses the peak spike velocity, $v_{\max }^{s}$, as the experimental metric to compare with the model [22]. By contrast, the originally proposed method to quantitatively estimate strength used the total integrated spike growth as the experimental metric, but that method was based on calculations for a general RMI configuration with a perturbed interface between two materials [18]. In our more limiting case with perturbations on the free surface, local interactions of release waves from the perturbed surface eventually result in tensile stresses leading to damage in the form of porosity for a ductile metal $[38,39]$. In previous work, it was demonstrated that the peak spike velocity occurred early enough to be unaffected by porosity, and it still has excellent sensitivity to strength; hence it makes an ideal metric [22]. Soon after the peak and well before the spike has arrested, the porosity grows enough to affect the total spike growth and compromise a strength estimate based on this total growth.

The RMI experiments were modeled using FLAG, an arbitrary Lagrangian-Eulerian, explicit, finite-volume hydrodynamics code using arbitrary polyhedral computational zones (elements) [40-44], which has been used for modeling many physical phenomena [45-51]. No mesh relaxation or Eulerian remapping was used in these calculations because all the spikes arrested in these RMI experiments leading to only modest deformations. The volumetric behavior of tantalum was modeled using a tabular SESAME equation of state [52-54]. Because the tantalum in these experiments stays solid in the ambient bcc $\alpha$ phase, essentially similar behavior can also be achieved using a Mie-Grüneisen equation of state with a linear $U_{s}-U_{p}$ relation. A temperature- and density- (i.e., pressure) 


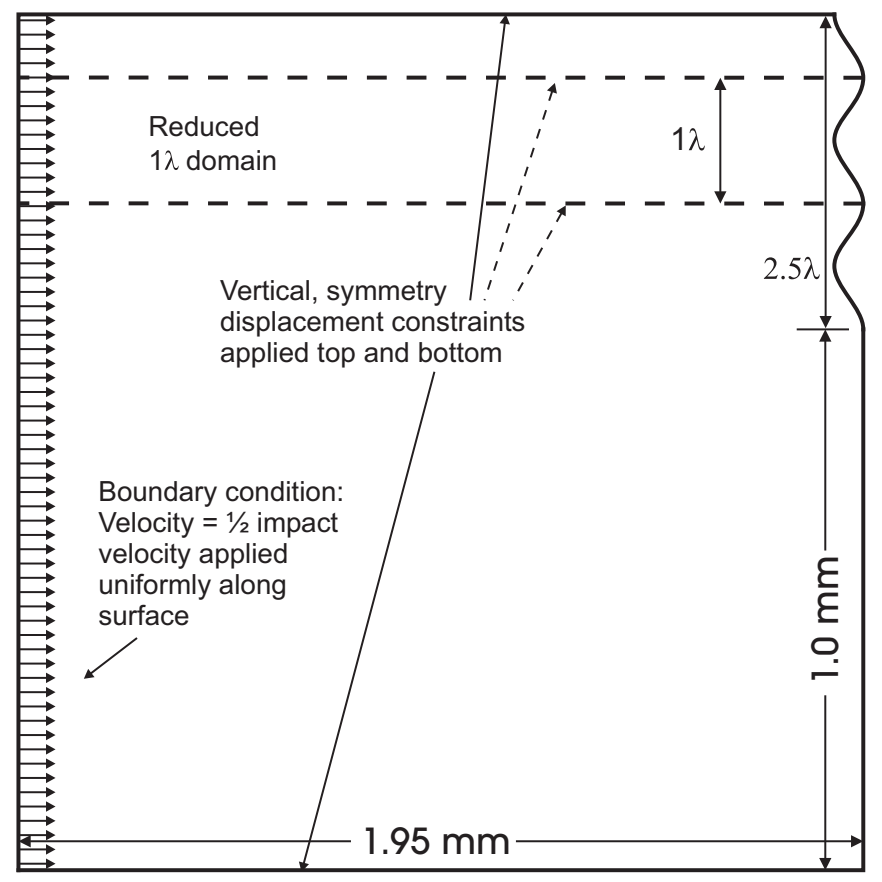

FIG. 6. The reduced computational domains model only the target and use a velocity boundary condition to model the impact load. The larger outer domain is a half symmetry model of the 5 -wavelength perturbation region and adjacent flats. The simpler, 1-wavelength reduced domain models the behavior of an infinitely repeating perturbation. (Approximately to scale for $\eta_{0} k=0.8$.)

dependent shear modulus treatment was used [55-57]. For reference in case such a model is not available, simpler calculations using merely the ambient-pressure temperaturedependent shear modulus caused slight changes to the sound wave speeds, which changed the spike velocities and then final strength estimate by only about $2 \%$. The constant strength model, i.e., elastic-perfectly plastic, used $J_{2}$ plasticity and the associated flow rule.

The computations used a two-dimensional plane strain mesh on a cross section through the perturbation. Figure 6 shows the two computational domains studied. A smaller $1 \lambda$ domain with symmetry boundary conditions, which effectively assumes infinitely repeating perturbations, was used for strength estimation. A larger domain including half of the full $5 \lambda$ perturbation region and half of the flat region between perturbation regions was used to check the validity of using the $1 \lambda$ domain. Because the impactor used the same tantalum as the target, the model used a velocity boundary condition of half the impact velocity on the impact surface rather than explicitly model the impactor. That assumption suffers no loss of accuracy but is valid only until the release wave off the free surface returns to the impact surface. Some additional calculations that included the impactor confirmed that the release wave does not perturb the velocities of the perturbations until well after the time of interest.

As discussed in detail in previous work [22], numerical viscosity in the simulations has an artificial damping effect on the velocity of the perturbations and can lead to significant errors in strength estimates. The calculations here used a classic VonNeumann and Richtmyer (VNR) viscosity approach

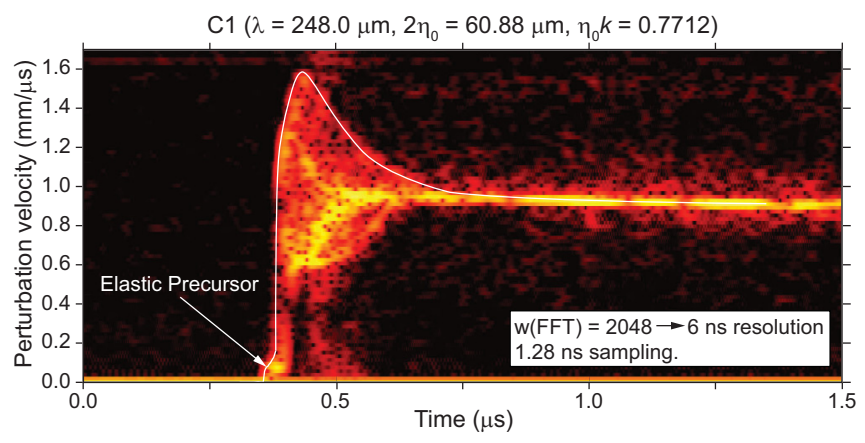

FIG. 7. A typical PDV spectrogram shows a spectrum of velocities corresponding to spikes, the original high spots that are inverting, and the regions between. A white line shows the approximate history of the spike velocity. The spectrogram is colored by intensity from the Fourier transform from black at the lowest intensity to red, yellow, and white (black to gray to white in print version). Because of the complicated interplay of the detector and the light reflecting off the angled surface, the intensity magnitudes have no significant interpretive value.

with a quadratic coefficient of 1.2 and a linear coefficient of 0.15 [58]. The viscosity effect converges away, so the strength estimates used peak spike velocities from meshes with zone sizes of $\lambda / 40, \lambda / 80$, and $\lambda / 160$ and then a linear best fit of those three values extrapolated to a zero zone size. A total of 378 calculations were required to cover the three experiments, six perturbations regions, three zones sizes, and seven Y's from 800 to $2000 \mathrm{MPa}$ in increments of 200.

Since porosity from tensile-stress-induced damage is always a concern for data interpretation, the Tonks ductile amage model as calibrated on tantalum [59-62] was used to confirm that porosity did not grow early enough to affect the peak spike velocity in these experiments.

\section{RESULTS AND DISCUSSION}

\section{A. Experimental results}

Figure 7 shows a typical PDV spectrogram from a probe interrogating a perturbation region, this one for the $\eta_{0} k=$ 0.77 region of the $30 \mathrm{GPa}$ experiment. Several features are visible. First, a small elastic precursor is visible as was the case at all three shock levels. After the precursor, the time between the arrival of the shock wave at the perturbation minimum, to its arrival at the perturbation maximum, is about $15 \mathrm{~ns}$, which is $2 \eta_{0} / U_{S}$, where $U_{S} \approx 4 \mathrm{~mm} / \mu \mathrm{s}$. This is more obvious on the images in Fig. 8 as the difference between the vertical solid red and dashed green lines that follow the small precursors that appear first in the spike region (solid red line), and then in the bubble region (dashed green line). Over approximately $400 \mathrm{~ns}$ from shock arrival, the evolution of the instabilities is captured in the Fig. 7 spread of velocities. The highest velocities correspond to the low perturbation region evolving into a spike; see Fig. 1. From the approximately 1 $\mathrm{mm} / \mu$ s peak particle velocity of the shock, the spike velocity rises over the course of about $70 \mathrm{~ns}$ to its peak value near $1.6 \mathrm{~mm} / \mu \mathrm{s}$. Roughly $300 \mathrm{~ns}$ later, the spike velocities and the lower velocities corresponding to the original high spots that are starting to invert all come back together, indicating spike 


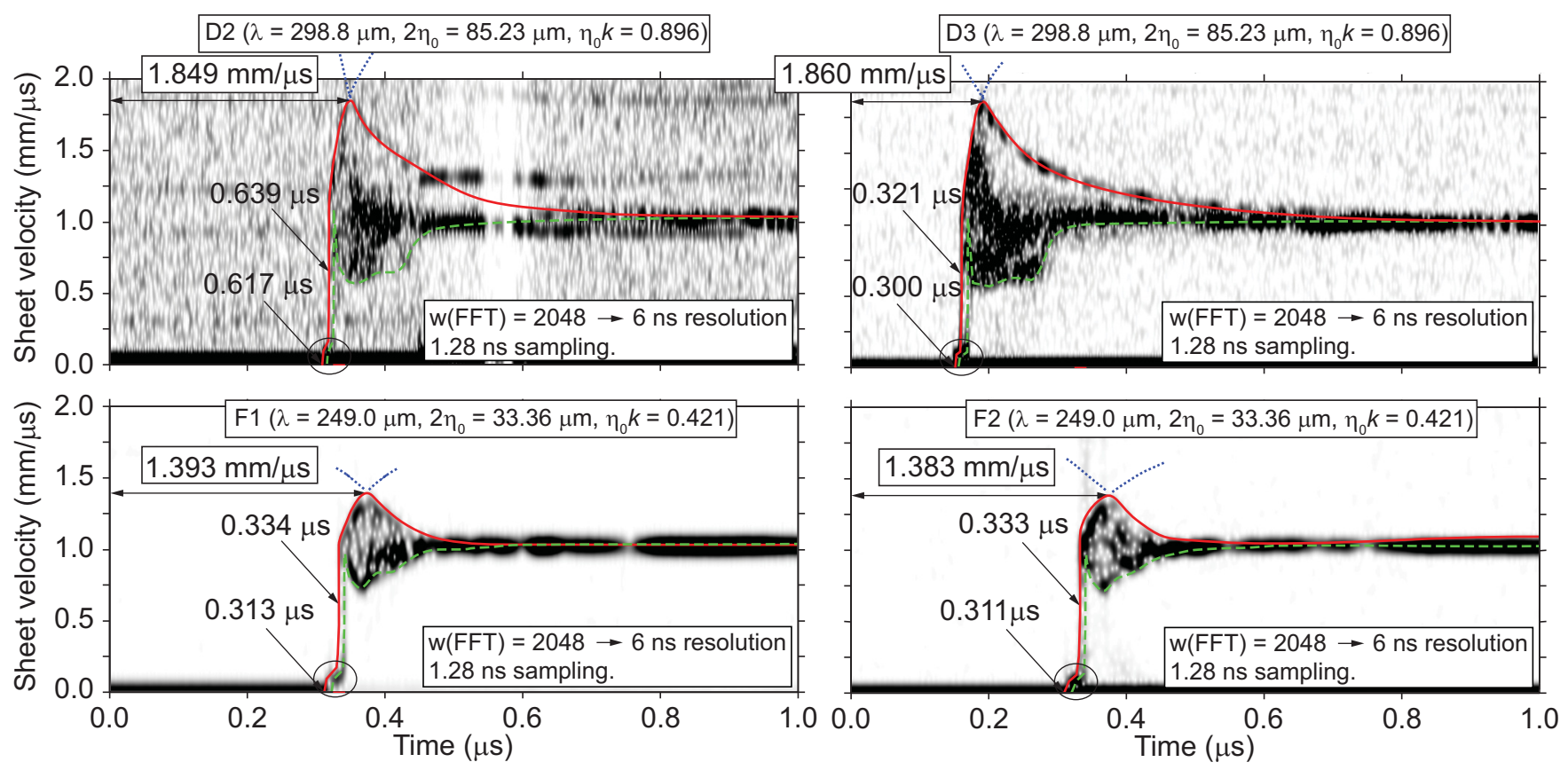

FIG. 8. For the $34 \mathrm{GPa}$ experiment, two velocity spectrograms each from perturbation zones D and F, with $k h_{0}=0.896$ and 0.421 , respectively. The upper two images are examples of difficult extractions to analyze, and the lower two images are examples of easier extractions to analyze. The final extracted peak velocities reported in this manuscript are indicated on the figure.

arrest. In these experiments, all of the perturbation regions at all three shock levels arrested.

Peak spike velocities must be extracted in order to estimate strength, but a distinct spike velocity and its peak are not always evident in the PDV spectrograms. Therefore, the data are considered collectively with an understanding of the physical phenomena. Figure 8 illustrates the process with example spectrograms from the $34 \mathrm{GPa}$ experiment. The titles at the tops of the images includes the probe labels for the perturbation zones; refer to Fig. 2. Distinct elastic precursor and shock wave arrivals at the perturbation minima and maxima are present in the velocity spectra. The earlier shock wave corresponding to the spike is identified from the raw data signal, located on the spectrogram and a spline from the end of the precursor is added to the image. This spline is fitted upward from the end of the peak precursor velocity and tends to show an inflection about halfway between the peak precursor velocity and the impact velocity. From the impact velocity the spike velocity rises sharply and rolls over toward the peak velocity. The spline is extended beyond the peak along its last slope, shown as a dotted blue line in Fig. 8. Once this spline is in place, another spline is fitted from the right along the shape of the decelerating growth, which is asymptotic to the impulse velocity. This arrested growth spline extends to the left of the growth phase spline into a $\mathrm{V}$ pattern above the sheet velocity, shown as another dotted blue line. Once the two splines are in place, they are cut, joined, and smoothed to a rounded peak with a third order polynomial spline; the peak sheet velocity will be less than the peak velocity defined by the intersection of the splines and the $\mathrm{V}$, and it will be greater than or equal to the rounded peak of the joined splines. Simulations further support this understanding of the spike-velocity time history. The reported peak spike velocity is the rounded spline velocity peak, with uncertainty estimates. It should be noted that the D2 spectrogram in Fig. 8 was the only perturbation velocity frequency-multiplexed in the recording system, which was done to add channels. It is the noisiest of all the signals and has some multiplexing artifacts like the flat line at $1.25 \mathrm{~mm} / \mu \mathrm{s}$ that falsely makes it look like the spike did not arrest. The other coverage on that perturbation, like D3, gave cleaner data.

Figure 9 shows the peak spike velocities extracted from the PDV spectrograms for all three experiments. The velocities

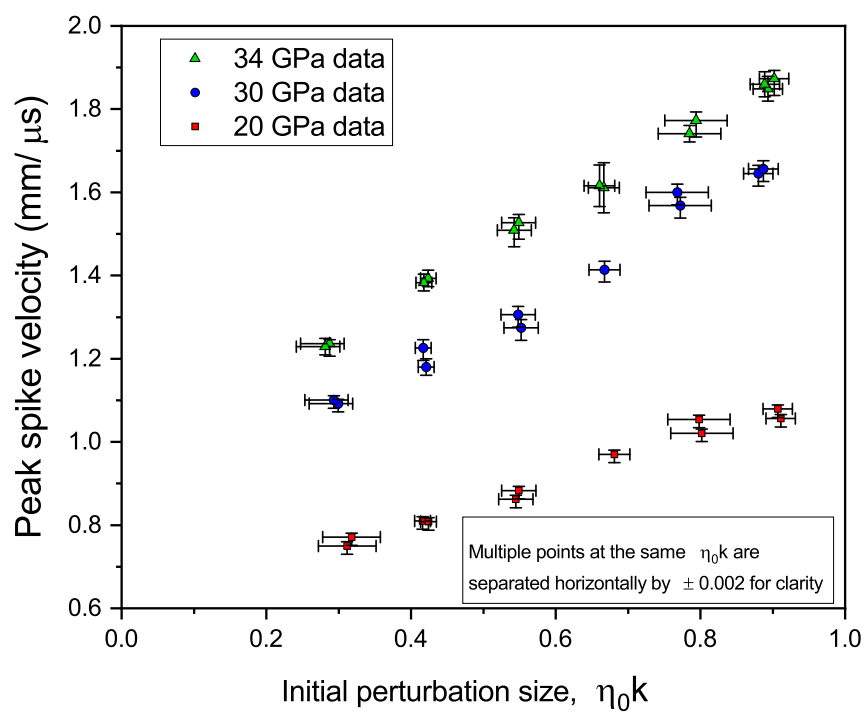

FIG. 9. The peak spike velocities extracted from the PDV spectrograms show the expected trends of velocities increasing steadily with increasing initial perturbation size. The multiple points for a given $\eta_{0} k$ correspond to the multiple velocimetry points in Fig. 2. 
TABLE I. Impact velocities, as-built perturbation geometries, and measured peak spike velocities.

\begin{tabular}{|c|c|c|c|c|c|c|c|c|c|}
\hline \multirow[b]{2}{*}{$\begin{array}{c}N o m \\
\eta_{0} k\end{array}$} & \multicolumn{3}{|c|}{$\begin{array}{c}20 \mathrm{GPa} \text { shot } \\
v_{\text {impact }}=642 \mathrm{~m} / \mathrm{s}\end{array}$} & \multicolumn{3}{|c|}{$\begin{array}{c}30 \mathrm{GPa} \text { shot } \\
v_{\text {impact }}=916 \mathrm{~m} / \mathrm{s}\end{array}$} & \multicolumn{3}{|c|}{$\begin{array}{c}34 \mathrm{GPa} \text { shot } \\
v_{\text {impact }}=1007 \mathrm{~m} / \mathrm{s}\end{array}$} \\
\hline & $\begin{array}{l}\text { Actual } \\
\lambda(\mu \mathrm{m})\end{array}$ & $\begin{array}{c}\text { Actual } \\
\eta_{0} k\end{array}$ & $\begin{array}{c}v_{\max }^{s} \\
(\mathrm{~mm} / \mu \mathrm{s})\end{array}$ & $\begin{array}{l}\text { Actual } \\
\lambda(\mu \mathrm{m})\end{array}$ & $\begin{array}{c}\text { Actual } \\
\eta_{0} k\end{array}$ & $\begin{array}{r}v_{\max }^{s} \\
(\mathrm{~mm} / \mu \mathrm{s})\end{array}$ & $\begin{array}{l}\text { Actual } \\
\lambda(\mu \mathrm{m})\end{array}$ & $\begin{array}{c}\text { Actual } \\
\eta_{0} k\end{array}$ & $\begin{array}{c}v_{\max }^{s} \\
(\mathrm{~mm} / \mu \mathrm{s})\end{array}$ \\
\hline 0.30 & 249.5 & $\begin{array}{l}0.315 \\
\pm 0.04\end{array}$ & $\begin{array}{l}0.771+0.01-0.02 \\
0.750+0.01-0.02\end{array}$ & 248.6 & $\begin{array}{c}0.296 \\
+0.02-0.04\end{array}$ & $\begin{array}{l}1.092+0.01-0.02 \\
1.101+0.01-0.02\end{array}$ & 246.3 & $\begin{array}{c}0.284 \\
+0.02-0.04\end{array}$ & $\begin{array}{l}1.236+0.01-0.03 \\
1.229+0.02-0.02\end{array}$ \\
\hline 0.42 & 249.0 & $\begin{array}{c}0.420 \\
\pm 0.011\end{array}$ & $\begin{array}{l}0.810+0.01-0.02 \\
0.808+0.01-0.02\end{array}$ & 249.2 & $\begin{array}{c}0.419 \\
\pm 0.011\end{array}$ & $\begin{array}{l}1.226+0.01-0.03 \\
1.180+0.02-0.02\end{array}$ & 249.0 & $\begin{array}{c}0.421 \\
\pm 0.011\end{array}$ & $\begin{array}{l}1.393+0.02-0.02 \\
1.383+0.02-0.02\end{array}$ \\
\hline 0.54 & 248 & $\begin{array}{c}0.547 \\
\pm 0.024\end{array}$ & $\begin{array}{l}0.862+0.01-0.02 \\
0.881+0.01-0.02\end{array}$ & 248.3 & $\begin{array}{c}0.550 \\
\pm 0.024\end{array}$ & $\begin{array}{l}1.306+0.02-0.03 \\
1.274+0.02-0.03\end{array}$ & 248.5 & $\begin{array}{c}0.546 \\
\pm 0.024\end{array}$ & $\begin{array}{l}1.527+0.02-0.04 \\
1.509+0.03-0.04\end{array}$ \\
\hline 0.66 & 247.5 & $\begin{array}{c}0.681 \\
\pm 0.021\end{array}$ & $0.970+0.01-0.02$ & 247.7 & $\begin{array}{c}0.667 \\
\pm 0.021\end{array}$ & $1.414+0.02-0.03$ & 247.5 & $\begin{array}{c}0.663 \\
\pm 0.021\end{array}$ & $\begin{array}{l}1.611+0.06-0.06 \\
1.616+0.05-0.05\end{array}$ \\
\hline 0.78 & 247.3 & $\begin{array}{c}0.800 \\
\pm 0.043\end{array}$ & $\begin{array}{l}1.054+0.01-0.02 \\
1.021+0.01-0.02\end{array}$ & 248.0 & $\begin{array}{c}0.771 \\
\pm 0.043\end{array}$ & $\begin{array}{l}1.600+0.02-0.03 \\
1.568+0.02-0.03\end{array}$ & 245.5 & $\begin{array}{c}0.789 \\
\pm 0.043\end{array}$ & $\begin{array}{l}1.773+0.02-0.04 \\
1.741+0.02-0.02\end{array}$ \\
\hline 0.90 & 297.3 & $0.909 \pm 0.020$ & $\begin{array}{r}1.079+0.01-0.02 \\
1.056+0.01-0.02\end{array}$ & 296.0 & $0.883 \pm 0.020$ & $\begin{array}{r}1.645+0.02-0.03 \\
1.656+0.02-0.03\end{array}$ & 298.8 & $0.896 \pm 0.020$ & $\begin{array}{c}1.873+0.02-0.04 \\
1.849 \pm 0.03 \\
1.860 \pm 0.03\end{array}$ \\
\hline
\end{tabular}

increase as $\eta_{0} k$ increases and as the shock stress increases. The data from separate probes for a given perturbation region agree within uncertainty. Table I gives the impact velocities, values for the peak spike velocities, and the as-built geometry determined from inspection.

\section{B. Assumption on computational domain}

Spike velocities from calculations with the larger computational domain from Fig. 6 are compared in Fig. 10 with those from the smaller domain. The results are plotted for the $\eta_{0} k=0.883$ case from the $30 \mathrm{GPa}$ experiment but are typical. The spike nearest the flat region, labeled spike 1, shows a slightly higher peak spike velocity than the other spikes. Until later in time, the curves for the two central spikes, which by symmetry represent the three central spikes in the experiment,

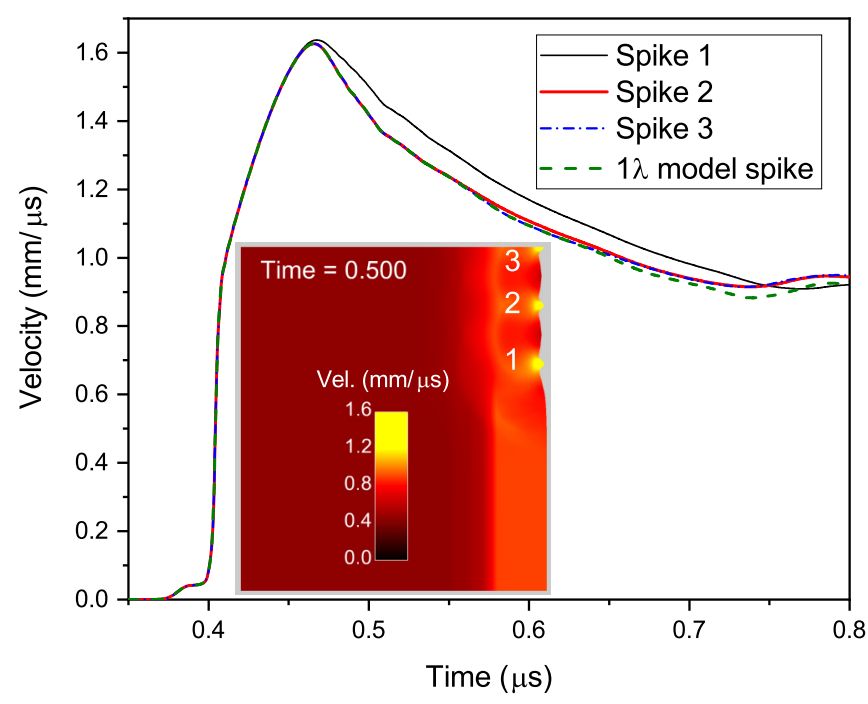

FIG. 10. The reduced domain $1 \lambda$ model is sufficient to capture the peak spike velocity for the central spikes in the perturbation region. are indistinguishable from the spike velocity from the reduced $1 \lambda$ domain calculation. Since the spot size and alignment for the PDV probes ensure that the velocity is only measured for the three central spikes, the $1 \lambda$ domain calculations were used for calculating peak spike velocity for strength estimation.

Since planar one-dimensional (1D) shock response is much less sensitive to strength than the perturbations, the velocities measured in flat regions were used to validate the impact velocity and Ta equation of state. Unfortunately, calculations with the larger domain showed that the velocity measured in the center of the flat region is affected by the nearest perturbations as early as $200 \mathrm{~ns}$ after shock breakout. Such a short duration of validity for the 1D approximation somewhat limits the validation exercise, especially since strength effects prevent a sharp rise to the final particle velocity within the 200 ns. Nevertheless, the 1D models reproduced all the available flat data to within the roughly $1.5 \%$ repeatability of those data.

\section{Strength estimate}

Figure 11 shows the comparison between the converged, elastic-perfectly plastic model results and the data of Fig. 9. For each constant strength value used in the calculations at each shock level, the root-mean-square average misfit between the predicted spike velocities and the measured values over all six perturbation sizes was calculated. The 20 and $30 \mathrm{GPa}$ shots show a minimum around $0.02 \mathrm{~mm} / \mu \mathrm{s}$, or about $2 \%$ and $1 \%$, respectively, of the peak velocities for the largest perturbation size. The $34 \mathrm{GPa}$ experiment shows a minimum of about $0.036 \mathrm{~mm} / \mu \mathrm{s}$, or about $2 \%$.

The curves in Fig. 11 were interpolated to find precise $Y$ 's that minimize the misfit. The $v_{\max }^{s}$ values were then interpolated similarly and are plotted in Fig. 12 and compared to the data. In general, the estimated strengths fit the data quite well. Uncertainties in the strength values were estimated by considering the uncertainties on individual data points and the ability of the strength to simultaneously fit all the data. Peak spike velocities corresponding to the uncertainty ranges in strengths were then calculated and are plotted as dashed 


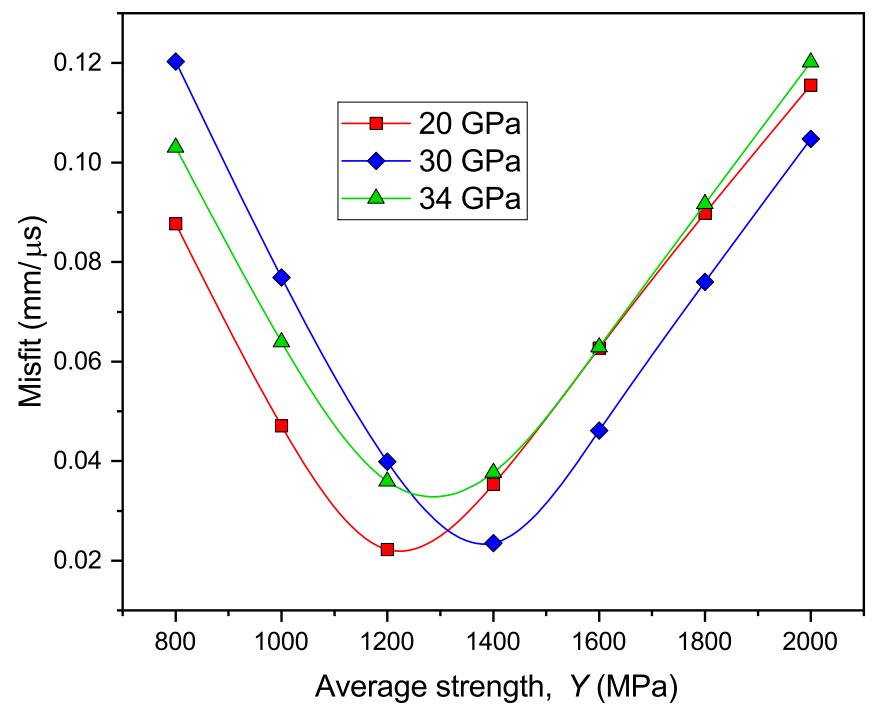

FIG. 11. Minima in the root-mean-square misfit between calculations and data indicate the average strength that best matches the data.

lines in Fig. 12. Uncertainties in $Y$ range from about $7 \%$ for the 20 and $30 \mathrm{GPa}$ data to $12 \%$ for the $34 \mathrm{GPa}$ data. The larger uncertainty for the $34 \mathrm{GPa}$ experiment is consistent with the larger misfit in Fig. 11. The uncertainties in Fig. 12 are intended to capture random uncertainties. The largest expected potential bias error would be uncertainty in the impact velocity. Additional calculations indicated that a $1 \%$ change in the impact velocity, about the experimental uncertainty, would result in a roughly $7 \%$ change in the strength estimate. Such a bias error would affect each shock level independently.

Recall that the largest perturbation size for each experiment $\left(\eta_{0} k=0.9\right)$ had a slightly larger wavelength: $300 \mu \mathrm{m}$ compared to $250 \mu \mathrm{m}$. Figure 12 shows that those data points fall slightly below the trend lines from the model fit. However, the deviations are mostly insignificant compared to the uncertain-

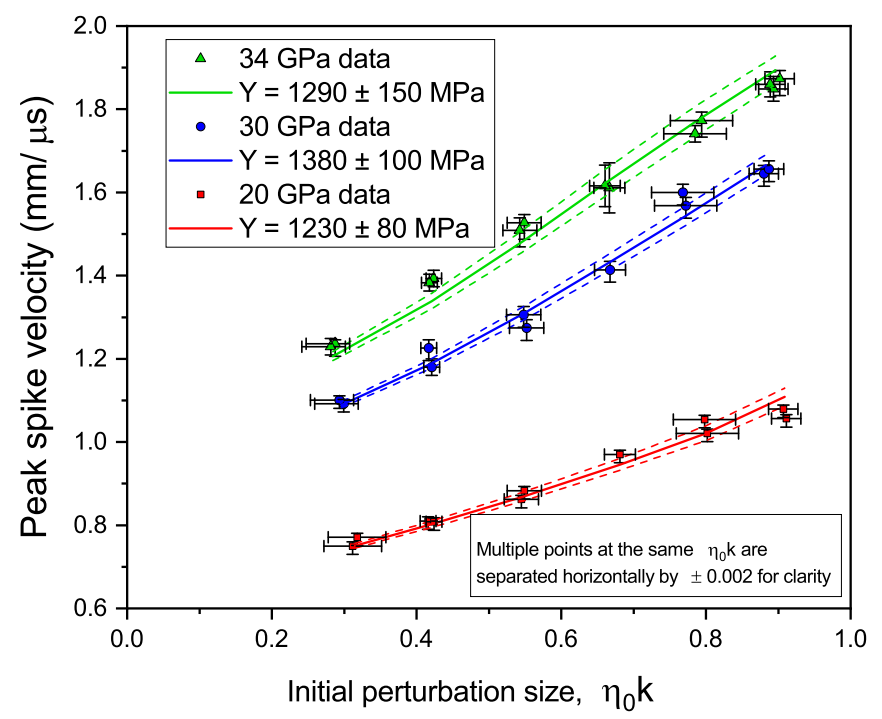

FIG. 12. Model-based average strength estimates fit the measured data quite well. ties. Further experiments are planned to check the consistency of RMI strength estimates over a wide range of perturbation wavelengths.

Simulations using the best fit $Y$ 's helped to put the strength estimates into the context of important physical quantities that affect strength: strain, strain rate, and temperature. As an example, Fig. 13 shows results from the $1 \lambda$ model calculation for the largest perturbation size on the $30 \mathrm{GPa}$ shock experiment with $Y$ set to $1380 \mathrm{MPa}$ per Fig. 12. The simulated spike velocity matches the PDV data quite well until just after the peak velocity. After that time, two effects cause the spike to arrest much more quickly (the velocity returns to the impact velocity) in the model. First, the strain rate falls off rapidly [22] which should make the actual strength decrease, whereas the simulation has a constant strength. Second, porosity growth in the experiment becomes sufficient to further reduce the strength. Figure 13(b) shows the shape of the perturbation at the time of the peak spike velocity and is colored by effective plastic strain. This experiment had relatively large growth because of the large perturbation size, yet the spike growth at this time is modest compared to the notional RMI illustration in Fig. 1. The peak plastic strain in the spike is still significant at nearly $100 \%$.

Following the work of [22], the computation zone with the highest plastic strain at the time of the peak spike velocity, which is several zones subsurface in the spike, is taken as the most representative of the conditions influencing the strength estimate. Along with the strength estimates, Table II shows the values extracted from the simulations. The plateau in strain rate after the shock, which is taken as representative for the strength estimate (see Fig. 16 in [22]), varies by about a factor of 4 from the smallest to largest perturbation sizes for a given shot and only about a factor of 2 from the $20 \mathrm{GPa}$ shot to the $34 \mathrm{GPa}$ shot. Considering that strength tends to vary with the log of the strain rate, these differences in strain rate are not very significant. Because $v_{\max }^{s}$ is used to estimate strength, behavior after the time of $v_{\max }^{s}$ is not relevant, so accumulated plastic strain and temperature are reported at the time of $v_{\max }^{s}$. Since the strength estimate encompasses integrated behavior up to that time, the most representative value should be some type of average, but the final value is reported for reference. The plastic strain varies by a factor of up to 5 between the smallest and largest perturbation size for a given shock, and less than a factor of 2 from the $20 \mathrm{GPa}$ shot to the $34 \mathrm{GPa}$ shot. Since tantalum shows only modest strain hardening at high rates, the strength differences from the different amounts of plastic strain are also expected to be modest. The final temperatures include the residual heating from the shock and also adiabatic heating from plastic work and so show similar trends as the plastic strain.

It would be possible to analyze the data differently. As was done previously, the current analysis groups all six perturbation sizes from each impact experiment together to give one strength estimate per experiment [22]. A strength estimate was also attempted for each individual perturbation region. With the data uncertainties in Fig. 12, the uncertainties on individual strengths were large and no informative trends were observed. With more precise experiments in the future, strength estimates for each individual perturbation might be useful. For now, grouping them together provides a more 


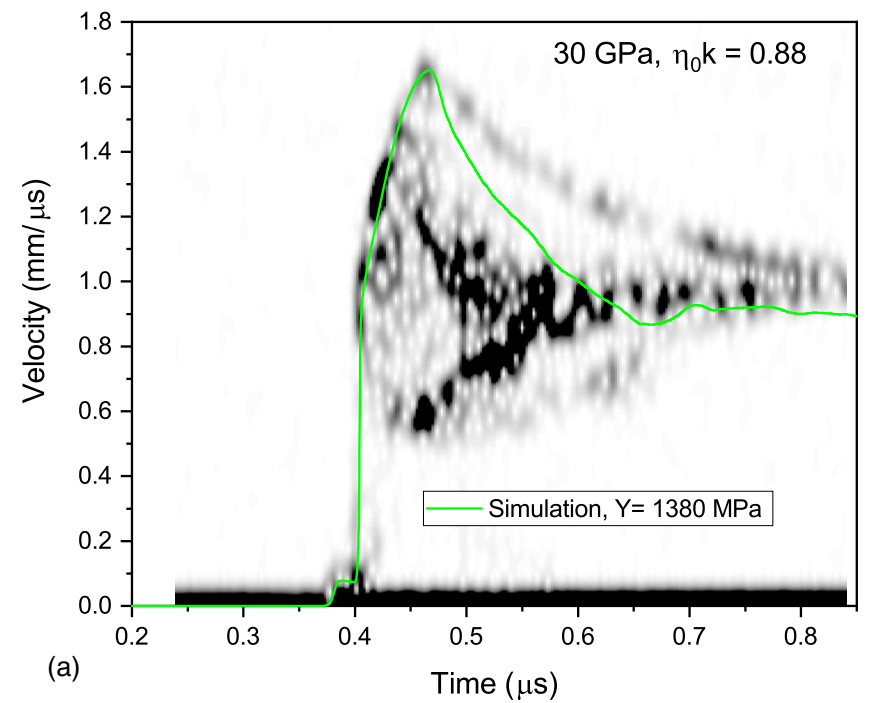

FIG. 13. A simulation of the $\eta_{0} k=0.883$ region for the $30 \mathrm{GPa}$ experiment using $Y=1380 \mathrm{MPa}$. (a) The simulated velocity matched the PDV spectrogram until the peak. (b) At the time of $\boldsymbol{v}_{\max }^{s}$, peak plastic strains are almost $100 \%$.

precise estimate, assuming random uncertainties. Based on Table II, one might group the data by strain rate or some criteria other than the impact pressure. However, because impact velocity is a significant uncertainty source that will vary between experiments but not within one experiment, combining data from different experiments might decrease precision.

\section{In context with other Ta strength measurements}

Even beyond the regime accessible by quasistatic and Hopkinson bar techniques, there is still substantial literature on the strength of tantalum, including instability-based experiments [26,63-65], shock-based measurements [66-68], and ramp loading experiments $[69,70]$ all at high rates and high pressure, and diamond anvil cell measurements [71-73] and other novel techniques [74] at low rate and high pressure. The results are not always consistent with one another. To simplify discussion and remove the complications of material pedigree, processing, grain size, etc., comparisons in this section are limited to other measurements on the same batch of Starck tantalum.

Figure 14 shows the RMI strength estimates compared with other strength measurements at low pressure and varying strain rates. Because of the uncertainty levels and limited range in strain rates exercised by the shots, the three RMI strength estimates show no discernible trend with strain rate. Non-RMI data points are from quasistatic and Hopkinson bar testing of the same batch of tantalum. The strength was taken at $20 \%$ strain from those stress-strain curves. For the room temperature data, a power law (line on the log-log plot) is drawn through the points since that is known to well represent the Ta data up to rates of $10^{4} / \mathrm{s}$ [75]. For the RMI and Hopkinson bar testing, room temperature represents the starting temperature since adiabatic heating is expected. Other data at temperatures from 198 to $473 \mathrm{~K}$ are plotted for reference. Figure 14 also shows the high rate, phonon drag branch of the Preston-Tonks-Wallace (PTW) strength model as originally given for tantalum [76]. The RMI strength estimates noticeably exceed the power law trend extrapolated from lower rates, which is the common extrapolation. Because of shock heating in the RMI samples, comparing with the room temperature extrapolation likely underestimates the amount that the RMI strength exceeds model expectations. Since tantalum shows modest or negligible shock hardening [37], comparing RMI with data from unshocked samples seems plausible. The accelerated strain rate dependence observed in Fig. 14 is approximately consistent with strain rate hardening of Ta measured with mini-Kolsky bar measurements at rates up to $5 \times 10^{5} / \mathrm{s}$, although those measurements were made on finer grained Ta $[36,77]$. Note that more sophisticated strength models sometimes also predict rate hardening in excess of the power law extrapolation [78].

Capturing the RMI data with the high-rate PTW branch would require moving that branch in a full decade in strain

TABLE II. Estimated average strength and characteristic conditions taken from simulations. $T$ and $\varepsilon_{p}$ are given at $t$ of $v_{\max }^{s}$ in the zone in the spike with the highest plastic strain for the smallest and largest perturbations.

\begin{tabular}{lcccc}
\hline \hline & & At zone with max $\varepsilon_{\mathrm{p}}$ at time of peak spike velocity \\
\hline Shot $\mathrm{P}$ & $Y(\mathrm{MPa})$ & $\operatorname{Max} \varepsilon_{\mathrm{p}}$ & Plateau rate after shock $(/ \mathrm{s})$ & $T(\mathrm{~K})$ \\
\hline 20 & $1230 \pm 80$ & $0.18,0.67$ & $2.9 \times 10^{6}, 1.2 \times 10^{7}$ & 405,644 \\
30 & $1380 \pm 100$ & $0.20,0.96$ & $4.5 \times 10^{6}, 1.7 \times 10^{7}$ & 460,882 \\
34 & $1290 \pm 150$ & $0.32,1.24$ & $5.6 \times 10^{6}, 2.0 \times 10^{7}$ & 514,945 \\
\hline \hline
\end{tabular}




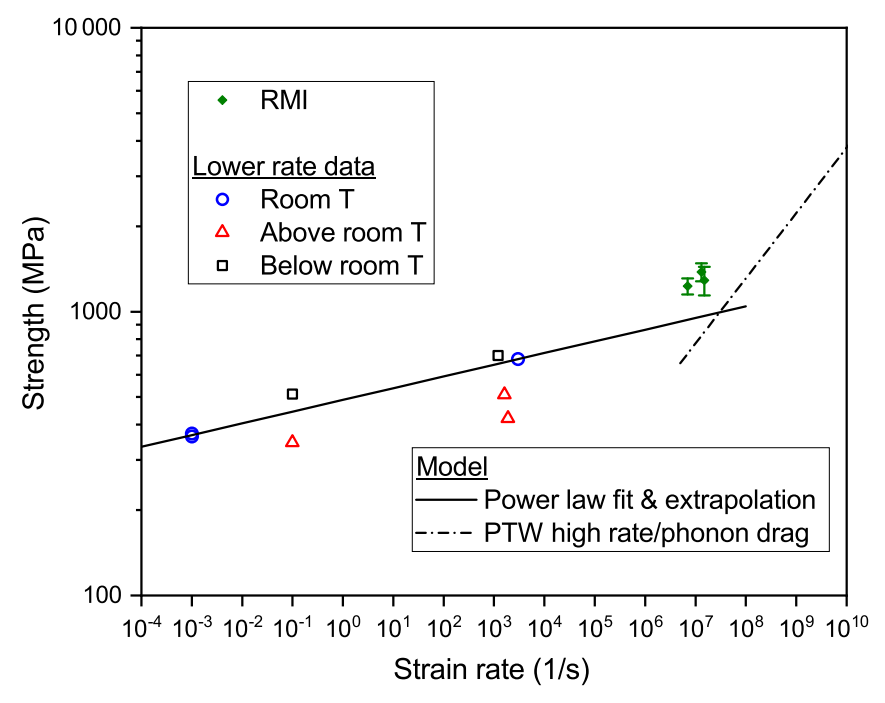

FIG. 14. The RMI data compared to other low pressure data starting from room temperature somewhat exceed the power law extrapolation.

rate, which cannot currently be justified. The RMI rates are in the five or more decade range in strain rates, between the Hopkinson bar calibration rates and the expected rate for the phonon drag strong shock regime, where strength models are mostly speculative [76]. These RMI results argue for a stronger transition between the two regimes for PTW and other similar models.

Figure 15 adds high-pressure strength measurements to the Fig. 14 data. Planar ramp loading and release experiments using magnetic loading were performed on the same batch of Starck tantalum at Sandia National Laboratories. A self-consistent Lagrangian analysis of time resolved velocity

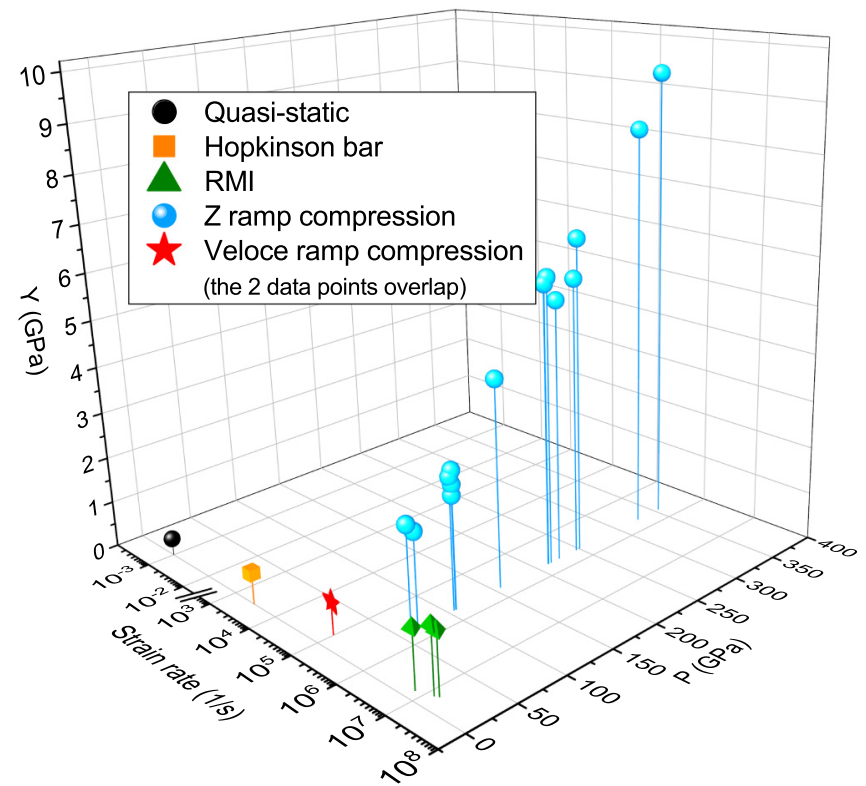

FIG. 15. The RMI data on tantalum shows modest rate hardening at rates up to $10^{7} / \mathrm{s}$ compared to apparently more significant pressure hardening. profiles measured during the experiments was used to estimate the mean shear stress near peak compression [79,80]. The data points in Fig. 15 include lower pressure experiments performed on the Veloce pulser [80] as well as previously published data from the $\mathrm{Z}$ machine [81] but with an updated analysis, and additional newer data points [82]. The data span pressures from 15 to $350 \mathrm{GPa}$ with strain rates all near $5 \times 10^{5} / \mathrm{s}$. In the context of this full data set, the RMI data are quite illuminating. The $\mathrm{Z}$ data show order-of-magnitude level increases in strength over lower pressure data, but prior to the RMI data it was difficult to establish how much of the strengthening came from rate effects and how much from pressure effects. With the RMI data at rates beyond those of the $\mathrm{Z}$ data showing modest strengthening over Hopkinson bar data, it appears that the pressure effects are more significant than the rate effects in this regime. Although it is difficult to extract a single strength estimate from them, and the samples used different tantalum, Rayleigh-Taylor experiments at 100 $\mathrm{GPa}$ and rates a bit above $10^{7} / \mathrm{s}$ support the trends in Fig. 15 [64].

\section{CONCLUSIONS AND FUTURE WORK}

Three Richtmyer-Meshkov instability experiments on tantalum gave average strength estimates of 1230-1380 MPa at estimated strain rates of $10^{7} / \mathrm{s}$. Estimated uncertainties range from $7 \%$ to $12 \%$. The use of impact loading, as compared to high explosive loading in previous experiments, made for simpler and more precise data analysis as well as the ability to explore a wider range of conditions. The measured strengths exceeded by $40 \%$ or more a power law extrapolation from data at strain rates below $10^{4} / \mathrm{s}$. The RMI experiments are currently unique in their ability to measure strength at such high rates but low pressures. Combined with experiments at high rates and high pressure, these data isolated rate effects on strength from pressure effects and indicated that, at least up to $10^{7} / \mathrm{s}$, the pressure effects are more significant.

The limiting factor in the precision and accuracy of the estimates seems to be extracting a peak spike velocity from the PDV velocity spectrograms. Continued development of experimental techniques is hoped to improve the quality of the PDV data. The range of shock stress in these experiments, 20-34 GPa, did not exercise a very broad range of strain rates. A broader range of shock stresses should be attempted, but faces practical constraints. To get similar behavior would require fabricating larger perturbations for lower stress shocks and smaller perturbations for larger stresses, which runs into machining limitations. In future experiments, the measurement of velocity in flat regions will be moved from between perturbation regions to the larger flat regions outside the outer perturbation regions. This move should provide a longer time record before nearby perturbations affect the velocity and therefore an improved validation of the model.

\section{ACKNOWLEDGMENTS}

This work was supported by the U.S. Department of Energy through the Los Alamos National Laboratory. Los Alamos National Laboratory is operated by Triad 
National Security, LLC, for the National Nuclear Security Administration of U.S. Department of Energy (Contract No. 89233218CNA000001). This work was supported by LANL's
Physics and Engineering Models (PEM) project of the Advanced Simulation and Computing Program (ASC) and the Dynamic Material Properties science campaign.
[1] K. O. Mikaelian, Effect of viscosity on Rayleigh-Taylor and Richtmyer-Meshkov instabilities, Phys. Rev. E 47, 375 (1993).

[2] P. Carlès and S. Popinet, The effect of viscosity, surface tension and non-linearity on Richtmyer-Meshkov instability, Eur. J. Mech., B: Fluids 21, 511 (2002).

[3] Y. Zhou, Rayleigh-Taylor and Richtmyer-Meshkov instability induced flow, turbulence, and mixing I, Phys. Rep. 720-722, 1 (2017).

[4] Y. Zhou, Rayleigh-Taylor and Richtmyer-Meshkov instability induced flow, turbulence, and mixing. II, Phys. Rep. 723-725, 1 (2017).

[5] A. Velikovich, M. Herrmann, and S. Abarzhi, Perturbation theory and numerical modelling of weakly and moderately nonlinear dynamics of the incompressible Richtmyer-Meshkov instability, J. Fluid Mech. 751, 432 (2014).

[6] K. O. Mikaelian, Richtmyer-Meshkov instability of arbitrary shapes, Phys. Fluids 17, 034101 (2005).

[7] J. N. Plohr and B. J. Plohr, Linearized analysis of RichtmyerMeshkov flow for elastic materials, J. Fluid Mech. 537, 55 (2005).

[8] A. R. Piriz, J. J. López Cela, N. A. Tahir, and D. H. H. Hoffmann, Richtmyer-Meshkov flow in elastic solids, Phys. Rev. E 74, 037301 (2006).

[9] K. O. Mikaelian, Shock-induced interface instability in viscous fluids and metals, Phys. Rev. E 87, 031003(R) (2013).

[10] A. López-Ortega, M. Lombardini, D. I. Pullin, and D. I. Meiron, Numerical simulations of the Richtmyer-Meshkov instability in solid-vacuum interfaces using calibrated plasticity laws, Phys. Rev. E 89, 033018 (2014).

[11] A. López-Ortega, M. Lombardini, P. T. Barton, D. I. Pullin, and D. I. Meiron, Richtmyer-Meshkov instability for elastic-plastic solids in converging geometries, J. Mech. Phys. Solids 76, 291 (2015).

[12] S. P. Rojas, E. N. Harstad, and R. G. Schmitt, Eulerian hydrocode estimates of Richtmyer-Meshkov instability growth and arrest, Procedia Eng 204, 367 (2017).

[13] G. Dimonte, G. Terrones, F. J. Cherne, and P. Ramaprabhu, Ejecta source model based on the nonlinear RichtmyerMeshkov instability, J. Appl. Phys. 113, 024905 (2013).

[14] A.-M. He, J. Liu, C. Liu, and P. Wang, Numerical and theoretical investigation of jet formation in elastic-plastic solids, J. Appl. Phys. 124, 185902 (2018).

[15] W. Georges, J. Loiseau, A. Higgins, and J. Zimmermann, Effect of scale, material strength, and loading on ejecta formation from explosively driven aluminum, J. Dyn. Behav. Mater. 3, 485. (2017).

[16] C. Roland, T. de Rességuier, A. Sollier, E. Lescoute, L. Soulard, and D. Loison, Hydrodynamic simulations of microjetting from shock-loaded grooves, in Shock Compression of Condensed Matter-2015, Tampa Bay, FL, Proceedings of the Conference of the American Physical Society Topical Group on Shock Compression of Condensed Matter, AIP Conf. Proc. No. 1793 (AIP, New York, 2017), p. 100027.
[17] A. R. Piriz, J. J. López Cela, N. A. Tahir, and D. H. H. Hoffmann, Richtmyer-Meshkov instability in elastic-plastic media, Phys. Rev. E 78, 056401 (2008).

[18] A. R. Piriz, J. J. L. Cela, and N. A. Tahir, Richtmyer-Meshkov instability as a tool for evaluating material strength under extreme conditions, Nucl. Instrum. Methods Phys. Res., Sect. A 606, 139 (2009).

[19] G. Dimonte, G. Terrones, F. J. Cherne, T. C. Germann, V. Dupont, K. Kadau, W. T. Buttler, D. M. Oro, C. Morris, and D. L. Preston, Use of the Richtmyer-Meshkov Instability to Infer Yield Stress at High-Energy Densities, Phys. Rev. Lett. 107, 264502 (2011).

[20] W. T. Buttler, D. M. Oró, D. L. Preston, K. O. Mikaelian, F. J. Cherne, R. S. Hixson, F. G. Mariam, C. Morris, J. B. Stone, G. Terrones, and D. Tupa, Unstable Richtmyer-Meshkov growth of solid and liquid metals in vacuum, J. Fluid Mech. 703, 60 (2012).

[21] B. J. Jensen, F. J. Cherne, M. B. Prime, K. Fezzaa, A. J. Iverson, C. A. Carlson, J. D. Yeager, K. J. Ramos, D. E. Hooks, J. C. Cooley, and G. Dimonte, Jet formation in cerium metal to examine material strength, J. Appl. Phys. 118, 195903 (2015).

[22] M. B. Prime, W. T. Buttler, M. A. Buechler, N. A. Denissen, M. A. Kenamond, F. G. Mariam, J. I. Martinez, D. M. Oró, D. W. Schmidt, J. B. Stone, D. Tupa, and W. Vogan-McNeil, Estimation of metal strength at very high rates using freesurface Richtmyer-Meshkov instabilities, J. Dyn. Behav. Mater. 3, 189 (2017).

[23] W. T. Buttler, G. T. Gray III, S. J. Fensin, M. Grover, M. B. Prime, G. D. Stevens, J. B. Stone, and W. D. Turley, Yield strength of $\mathrm{Cu}$ and a $\mathrm{CuPb}$ alloy $(1 \% \mathrm{~Pb})$, in Shock Compression of Condensed Matter-2015, Tampa Bay, FL, Proceedings of the Conference of the American Physical Society Topical Group on Shock Compression of Condensed Matter, AIP Conf. Proc. No. 1793 (AIP, New York, 2017), p. 110005.

[24] S. Opie, E. Loomis, P. Peralta, T. Shimada, and R. P. Johnson, Strength and Viscosity Effects on Perturbed Shock Front Stability in Metals, Phys. Rev. Lett. 118, 195501 (2017).

[25] Z. Sternberger, B. R. Maddox, Y. P. Opachich, C. E. Wehrenberg, R. G. Kraus, B. A. Remington, G. C. Randall, M. Farrell, and G. Ravichandran, A comparative study of RayleighTaylor and Richtmyer-Meshkov instabilities in 2D and 3D in tantalum, in Shock Compression of Condensed Matter-2015, Tampa Bay. FL, Proceedings of the Conference of the American Physical Society Topical Group on Shock Compression of Condensed Matter, AIP Conf. Proc. 1793 (AIP, New York, 2017), p. 110006.

[26] A. P. Stebner, C. E. Wehrenberg, B. Li, G. C. Randall, K. K. John, G. A. Hudish, B. R. Maddox, M. Farrell, H.-S. Park, and B. A. Remington, Strength of tantalum shocked at ultrahigh pressures, Mater. Sci. Eng.: A 732, 220 (2018).

[27] Z. Sternberger, Y. Opachich, C. Wehrenberg, R. Kraus, B. Remington, N. Alexander, G. Randall, M. Farrell, and 
G. Ravichandran, Investigation of hydrodynamic instability growth in copper, Int. J. Mech. Sci. 149, 475 (2018).

[28] P. Peralta, E. Loomis, Y. Chen, A. Brown, R. McDonald, K. Krishnan, and H. Lim, Grain orientation effects on dynamic strength of FCC multicrystals at low shock pressures: A hydrodynamic instability study, Philos. Mag. Lett. 95, 67 (2015).

[29] M. S. Freeman, C. Rousculp, D. Oro, S. Kreher, B. Cheng, J. Griego, A. Patten, L. Neukirch, R. Reinovsky, P. Turchi, J. Bradley III, W. Reass, F. Fierro, R. Randolph, P. Donovan, A. Saunders, F. Mariam, and Z. Tang, The spikes from RichtmyerMeshkov instabilities in pulsed power cylindrical experiments, in Shock Compression of Condensed Matter-2017, St. Louis, $M O$, Proceedings of the Conference of the American Physical Society Topical Group on Shock Compression of Condensed Matter, AIP Conf. Proc. No. 1979, edited by R. Chau, T. C. Germann, J. M. D. Lane, E. N. Brown, J. H. Eggert, and M. D. Knudson (AIP, New York, 2018), p. 080005.

[30] S. J. Vachhani, C. Trujillo, N. Mara, V. Livescu, C. Bronkhorst, G. T. Gray, and E. Cerreta, Local mechanical property evolution during high strain-rate deformation of tantalum, J. Dyn. Behav. Mater. 2, 511 (2016).

[31] T. E. Buchheit, E. K. Cerreta, L. Diebler, S.-R. Chen, and J. R. Michael, Characterization of Tri-lab Tantalum (Ta) Plate, Report No. SAND2014-17645, Sandia National Laboratories, 2014 (unpublished).

[32] H. Lim, H. J. Bong, S.-R. Chen, T. M. Rodgers, C. C. Battaile, and J. M. D. Lane, Developing anisotropic yield models of polycrystalline tantalum using crystal plasticity finite element simulations, Mater. Sci. Eng.: A 730, 50 (2018).

[33] O. T. Strand, D. R. Goosman, C. Martinez, T. L. Whitworth, and W. W. Kuhlow, Compact system for high-speed velocimetry using heterodyne techniques, Rev. Sci. Instrum. 77, 083108 (2006).

[34] W. T. Buttler, S. K. Lamoreaux, F. G. Omenetto, and J. R. Torgerson, Optical velocimetry, arXiv:Physics/0409073v1.

[35] M. B. Prime, Strain rate sensitivity of Richtmyer-Meshkov instability experiments for metal strength, in Dynamic Behavior of Materials, Vol. 1: Proceedings of the 2017 Annual Conference on Experimental and Applied Mechanics, edited by J. Kimberley, L. Lamberson, and S. Mates (Springer International Publishing, Cham, Switzerland, 2018), p. 13.

[36] D. Casem, D. Magagnosc, J. Ligda, B. Schuster, and T. Walter, Mechanical Behavior of Ta at Extreme Strain-Rates (Springer International Publishing, Cham, Switzerland, 2019), p. 195.

[37] D. H. Lassila and G. T. Gray III, Effects of shock prestrain on the dynamic mechanical behavior of tantalum, J. Phys. IV France 01, C3-19 (1991).

[38] J. Wang, F. Wu, Y. Zhu, A. He, Wang, and H. Wu, Unsupported shock wave induced dynamic fragmentation of matrix in lead with surface grooves, Comput. Mater. Sci. 156, 404 (2019).

[39] M. V. Antipov, V. A. Arinin, A. B. Georgievskaya, I. S. Gnutov, D. N. Zamyslov, D. A. Kalashnikov, M. O. Lebedeva, A. I. Lebedev, A. L. Mikhailov, V. A. Ogorodnikov, K. N. Panov, A. S. Pupkov, V. A. Rayevskiy, A. S. Sokolova, M. A. Syrunin, B. I. Tkachenko, A. A. Utenkov, A. V. Fedorov, S. A. Finyshin, and E. A. Chudakov et al., Experimental and computational damage and ejecta studies of $\mathrm{Pb}$ explosively shock loaded to $P_{S L} \approx 32$ - to $40-$ GPa, J. Dyn. Behav. Mater. 3, 300 (2017).
[40] E. J. Caramana, D. E. Burton, M. J. Shashkov, and P. P. Whalen, The construction of compatible hydrodynamics algorithms utilizing conservation of total energy, J. Comput. Phys. 146, 227 (1998).

[41] D. E. Burton, T. C. Carney, N. R. Morgan, S. R. Runnels, S. K. Sambasivan, and M. J. Shashkov, A cell-centered Lagrangian hydrodynamics method for multi-dimensional unstructured grids in curvilinear coordinates with solid constitutive models, Report No. LA-UR-11-04995, Los Alamos National Laboratory, 2011 (unpublished).

[42] M. Kenamond, M. Bement, and M. Shashkov, Compatible, total energy conserving and symmetry preserving arbitrary Lagrangian-Eulerian hydrodynamics in 2D rz-cylindrical coordinates, J. Comput. Phys. 268, 154 (2014).

[43] D. E. Burton, Connectivity structures and differencing techniques for staggered-grid free-Lagrange hydrodynamics, Lawrence Livermore National Laboratory, Report No. UCRLJC-110555, 1992 (unpublished).

[44] D. E. Burton, Consistent finite-volume discretization of hydrodynamic conservation laws for unstructured grids, Lawrence Livermore National Laboratory Report No. UCRL-JC-118788, 1994 (unpublished).

[45] J. Fung, A. K. Harrison, S. Chitanvis, and J. Margulies, Ejecta source and transport modeling in the FLAG hydrocode, Comput. Fluids 83, 177 (2013).

[46] S. K. Sambasivan, M. J. Shashkov, and D. E. Burton, A finite volume cell-centered Lagrangian hydrodynamics approach for solids in general unstructured grids, Int. J. Numer. Methods Fluids 72, 770 (2013).

[47] N. R. Morgan, M. A. Kenamond, D. E. Burton, T. C. Carney, and D. J. Ingraham, An approach for treating contact surfaces in Lagrangian cell-centered hydrodynamics, J. Comput. Phys. 250, 527 (2013).

[48] N. A. Denissen, B. Rollin, J. M. Reisner, and M. J. Andrews, The Tilted Rocket Rig: A Rayleigh-Taylor Test Case for RANS Models1, J. Fluids Eng. 136, 091301 (2014).

[49] K. Lipnikov, J. Reynolds, and E. Nelson, Mimetic discretization of two-dimensional magnetic diffusion equations, J. Comput. Phys. 247, 1 (2013).

[50] D. J. Luscher, M. A. Kenamond, A. Hunter, J. R. Mayeur, and H. M. Mourad, Implementation of a dislocation-density based single-crystal model into a continuum shock hydrodynamics code, in Shock Compression of Condensed Matter2017, St. Louis, MO, Proceedings of the Conference of the American Physical Society Topical Group on Shock Compression of Condensed Matter, AIP Conf. Proc. No. 1979, edited by R. Chau, T. C. Germann, J. M. D. Lane, E. N. Brown, J. H. Eggert, and M. D. Knudson (AIP, New York, 2018), p. 180006.

[51] D. J. Walters, A. Biswas, E. C. Lawrence, D. C. Francom, D. J. Luscher, D. A. Fredenburg, K. R. Moran, C. M. Sweeney, R. L. Sandberg, J. P. Ahrens, and C. A. Bolme, Bayesian calibration of strength parameters using hydrocode simulations of symmetric impact shock experiments of Al-5083, J. Appl. Phys. 124, 205105 (2018).

[52] S. P. Lyon and J. D. Johnson, SESAME: The Los Alamos National Laboratory equation of state database, Report No. LA-UR-92-3407, Los Alamos National Laboratory, 1992 (unpublished). 
[53] C. W. Greeff, S. D. Crockett, L. Burakovsky, and S. P. Rudin, Limited range SESAME EOS for Ta, Report No. LA-UR-1722600, Los Alamos National Laboratory, 2017 (unpublished).

[54] C. W. Greeff, S. P. Rudin, S. D. Crockett, and J. M. Wills, The cold equation of state of tantalum, in Shock Compression of Condensed Matter-2009, Nashville, TN, Proceedings of the Conference of the American Physical Society Topical Group on Shock Compression of Condensed Matter, AIP Conf. Proc. No. 1195, edited by M. Elert, M. D. Furnish, W. A. Anderson, W. G. Proud, and W. T. Butler (AIP, New York, 2009), p. 681.

[55] L. Burakovsky, C. W. Greeff, and D. L. Preston, Analytic model of the shear modulus at all temperatures and densities, Phys. Rev. B 67, 094107 (2003).

[56] L. Burakovsky, J. N. Plohr, S. K. Sjue, and D. J. Luscher, Thermoelasticity model for tantalum, Report No. LA-UR-1823823, Los Alamos National Laboratory, 2018 (unpublished).

[57] L. Burakovsky, D. J. Luscher, S. K. Sjue, D. E. Vaughan, and D. L. Preston, Generalization of the unified analytic melt-shear model to multi-phase materials: Molybdenum as an example, Crystals 9, 86 (2019).

[58] J. VonNeumann and R. D. Richtmyer, A method for the numerical calculation of hydrodynamic shocks, J. Appl. Phys. 21, 232 (1950).

[59] D. Tonks, A. Zurek, W. Thissell, J. Vorthman, and R. Hixson, The Tonks ductile damage model, Report No. LA-UR-03-0809, Los Alamos National Laboratory, 2002 (unpublished).

[60] A. K. Zurek, W. R. Thissell, J. N. Johnson, D. L. Tonks, and R. Hixson, Micromechanics of spall and damage in tantalum, J. Mater. Process. Technol. 60, 261 (1996).

[61] D. L. Tonks, Percolation wave propagation, and void linkup effects in ductile fracture, J. Phys. IV France 4, C8-665 (1994).

[62] D. L. Tonks, A. K. Zurek, and W. R. Thissell, Void coalescence model for ductile damage, in Shock Compression of Condensed Matter, 2001-Atlanta, GA, Proceedings of 12th APS Topical Conference, AIP Conf. Proc. No. 620, edited by M. D. Furnish, N. N. Thadhani, and Y. Horie (AIP, New York, 2002), p. 611.

[63] Z. Sternberger, B. Maddox, Y. Opachich, C. Wehrenberg, R. Kraus, B. Remington, G. Randall, M. Farrell, and G. Ravichandran, Inferring strength of tantalum from hydrodynamic instability recovery experiments, J. Dyn. Behav. Mater. 4, 244 (2018).

[64] H.-S. Park, N. Barton, J. L. Belof, K. J. M. Blobaum, R. M. Cavallo, A. J. Comley, B. Maddox, M. J. May, S. M. Pollaine, S. T. Prisbrey, B. Remington, R. E. Rudd, D. W. Swift, R. J. Wallace, M. J. Wilson, A. Nikroo, and E. Giraldez, Experimental results of tantalum material strength at high pressure and high strain rate, in Shock Compression of Condensed Matter2011, Chicago, IL, Proceedings of the Conference of the American Physical Society Topical Group on Shock Compression of Condensed Matter, AIP Conf. Proc. No. 1426, edited by M. E. Elert, W. T. Buttler, J. P. Borg, J. L. Jordan, and T. J. Vogler (AIP, New York, 2012), p. 1371.

[65] K. E. Duprey and R. J. Clifton, Pressure-shear response of thin tantalum foils, in Shock Compression of Condensed Matter1999, Snowbird, UT, AIP Conf Proc. No. 505, edited by M. D. Furnish, M. C. Chhabildas, and R. S. Hixson (AIP, New York, 2000), p. 447.
[66] A. J. Comley, B. R. Maddox, R. E. Rudd, S. T. Prisbrey, J. A. Hawreliak, D. A. Orlikowski, S. C. Peterson, J. H. Satcher, A. J. Elsholz, H.-S. Park, B. A. Remington, N. Bazin, J. M. Foster, P. Graham, N. Park, P. A. Rosen, S. R. Rothman, A. Higginbotham, M. Suggit, and J. S. Wark, Strength of ShockLoaded Single-Crystal Tantalum [100] Determined using In Situ Broadband X-Ray Laue Diffraction, Phys. Rev. Lett. 110, 115501 (2013).

[67] G. T. Gray III, N. K. Bourne, and J. C. F. Millett, Shock response of tantalum: Lateral stress and shear strength through the front, J. Appl. Phys. 94, 6430 (2003).

[68] M. D. Furnish, L. C. Chhabildas, and D. J. Steinberg, Dynamical behavior of tantalum, in High Pressure Science and Technology-1993, Colorado Springs, CO, AIP Conf. Proc. No. 309, edited by S. C. Schmidt, J. W. Shaner, G. A. Samara, and M. Ross (AIP, New York, 1994), p. 1099.

[69] J. H. Eggert, R. F. Smith, D. C. Swift, R. E. Rudd, D. E. Fratanduono, D. G. Braun, J. A. Hawreliak, J. M. McNaney, and G. W. Collins, Ramp compression of tantalum to $330 \mathrm{GPa}$, High Pressure Res. 35, 339 (2015).

[70] J. R. Asay, T. Ao, T. J. Vogler, J.-P. Davis, and G. T. Gray III, Yield strength of tantalum for shockless compression to $18 \mathrm{GPa}$, J. Appl. Phys. 106, 073515 (2009).

[71] A. Dewaele and P. Loubeyre, Mechanical properties of tantalum under high pressure, Phys. Rev. B 72, 134106 (2005).

[72] S. T. Weir, J. Akella, C. Ruddle, T. Goodwin, and L. Hsiung, Static strengths of Ta and U under ultrahigh pressures, Phys. Rev. B 58, 11258 (1998).

[73] Q. Jing, Q. Wu, J.-a. Xu, Y. Bi, L. Liu, S. Liu, Y. Zhang, and H. Geng, Anomalous softening of yield strength in tantalum at high pressures, J. Appl. Phys. 117, 055903 (2015).

[74] J. P. Escobedo, D. Field, D. Lassila, and M. Leblanc, A novel mechanical method to measure shear strength in specimens under pressure, MRS Symp. Proc. 929, 99 (2006).

[75] H. Lim, C. C. Battaile, J. L. Brown, and C. R. Weinberger, Physically-based strength model of tantalum incorporating effects of temperature, strain rate and pressure, Modell. Simul. Mater. Sci. Eng. 24, 055018 (2016).

[76] D. L. Preston, D. L. Tonks, and D. C. Wallace, Model of plastic deformation for extreme loading conditions, J. Appl. Phys. 93, 211 (2003).

[77] D. T. Casem, S. E. Grunschel, and B. E. Schuster, Normal and transverse displacement interferometers applied to small diameter Kolsky bars, Exp. Mech. 52, 173 (2012).

[78] N. R. Barton and M. Rhee, A multiscale strength model for tantalum over an extended range of strain rates, J. Appl. Phys. 114, 123507 (2013).

[79] J. R. Asay and J. Lipkin, A self-consistent technique for estimating the dynamic yield strength of a shock-loaded material, J. Appl. Phys. 49, 4242 (1978).

[80] J. L. Brown, C. S. Alexander, J. R. Asay, T. J. Vogler, and J. L. Ding, Extracting strength from high pressure ramp-release experiments, J. Appl. Phys. 114, 223518 (2013).

[81] J. L. Brown, C. S. Alexander, J. R. Asay, T. J. Vogler, D. H. Dolan, and J. L. Belof, Flow strength of tantalum under ramp compression to $250 \mathrm{GPa}$, J. Appl. Phys. 115, 043530 (2014).

[82] J. L. Brown, Sandia National Laboratories (private communication, July 2019). 